\title{
1 Design and test performance of the ATLAS Feature 2 Extractor trigger boards for the Phase-1 Upgrade
}

\section{Weiming Qian *}

$4 \quad$ STFC Rutherford Appleton Laboratory,

5 Chilton, Didcot, Oxon, OX11 0QX, United Kingdom

6 E-mail: Weiming.Qianestfc.ac.uk

7 ABSTRACT: In Run 3, the ATLAS Level-1 Calorimeter Trigger will be augmented by an 8 Electron Feature Extractor (eFEX), to identify isolated $e / \gamma$ and $\tau$ particles, and a Jet Feature 9 Extractor (jFEX), to identify energetic jets and calculate various local energy sums. Each 10 module accommodates more than 450 differential signals that can operate at up to $12.8 \mathrm{~Gb} / \mathrm{s}$, 11 some of which are routed over $30 \mathrm{~cm}$ between FPGAs. Presented here are the module designs, 12 the processes that have been adopted to meet the challenges associated with multi- $\mathrm{Gb} / \mathrm{s} \mathrm{PCB}$ 13 design, and the results of tests that characterize the performance of these modules.

14 KEYWORDS: Trigger; Feature Extractor; ATCA; PCB simulation.

*On behalf of ATLAS Collaboration 
1.1 ATLAS Level-1 Calorimeter Trigger architecture for Phase-I Upgrade

1.2 Trigger algorithms and performance 2

2. Prototype design $\quad 3$

$2.1 \mathrm{eFEX}$

2.1.1 Processing Area

2.1.2 eFEX prototype

$2.2 \mathrm{jFEX}$

2.2.1 Processing area

2.2.2 jFEX prototype

2.3 PCB design method

\section{Prototype test}

3.1 Link speed test results

3.2 Link speed decision

\section{Introduction}

In Run 3 (starting in 2021), the LHC [1] luminosity will double (to $\sim 2.5 \times 10^{34} \mathrm{~cm}^{-2} \mathrm{~s}^{-1}$ ), which will greatly increase the pileup rate. However, the ATLAS [2] front-end detector electronics will remain largely unchanged. Hence the total ATLAS Level-1 Trigger [3] rate will still be limited by the readout bandwidth of the front-end electronics to $100 \mathrm{KHz}$ or less. Moreover, the Level-1 Trigger must retain sensitivity to the physics electroweak processes and stay within the current ATLAS Level-1 latency envelope of $2.5 \mu \mathrm{s}$. To meet these challenges, the Phase-I Upgrade [4] to the ATLAS Level-1 Trigger system is needed.

\subsection{ATLAS Level-1 Calorimeter Trigger architecture for Phase-I Upgrade}

Figure 1 shows the architecture of the Phase-I Upgrade of the ATLAS Level-1 Calorimeter Trigger (L1Calo) [5]. The current L1Calo system is augmented by three additional featureidentification subsystems:

- the electromagnetic Feature Extractor (eFEX), comprising eFEX modules and Hub modules with Readout Driver (ROD) daughter cards, which identifies isolated e/ $\gamma$ and $\tau$ candidates, using data of finer granularity than is currently available to L1Calo;

- the jet Feature Extractor (jFEX), comprising jFEX modules and Hub modules with 
sums, using data of finer granularity than that available to the current L1Calo JEP subsystem;

- the global Feature Extractor (gFEX [6]), comprising one gFEX module, which identifies calorimeter trigger features requiring the complete calorimeter data.

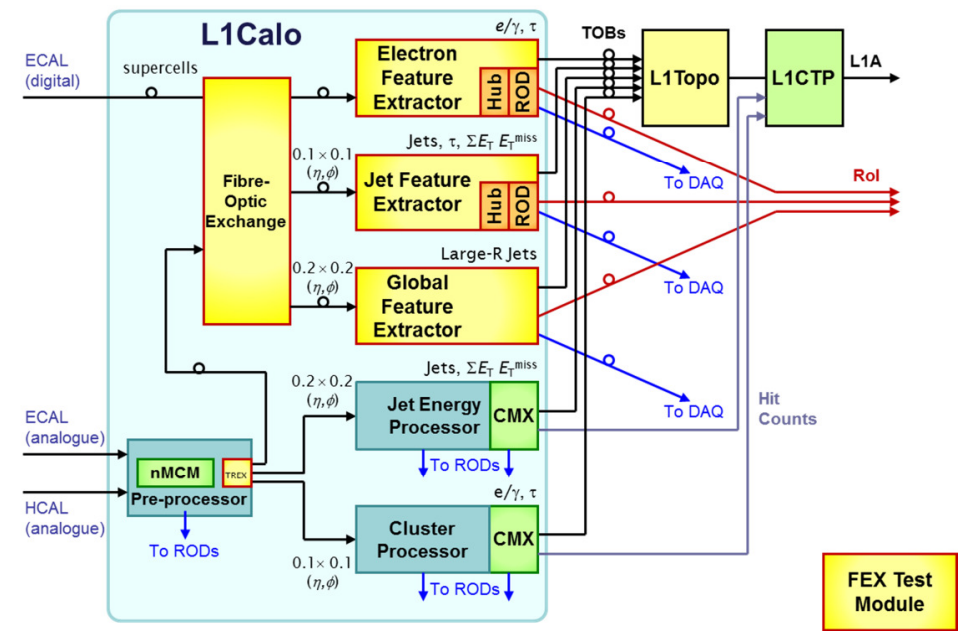

Figure 1. ATLAS Level-1 Calorimeter Trigger Phase-I Upgrade [7]

In addition to these, the Phase-I upgrade of L1Calo includes the Tile Rear Extension (TREX) to the Pre-Processor (PPr) subsystem, which digitizes Tile data and transmits them to the FEXs optically, the Fibre Optical Exchange (FOX), and the FEX Test Module (FTM), which facilitates the testing of FEX modules before system-level commissioning.

Apart from the small number of PPr modules that digitize Tile data for the FEXs, the current L1Calo system, comprising the Pre-Processor, Jet Energy Processor (JEP) and Cluster Processor (CP), will be decommissioned after the Phase-I Upgrade is fully commissioned.

\subsection{Trigger algorithms and performance}

In the current L1Calo system, the CP processes data from the calorimeters and identifies energy deposits characteristic of isolated $e / \gamma$ and $\tau$ particles, using Trigger Towers of typical granularity of $0.1 \times 0.1(\eta \times \phi)$. The eFEX performs this same function using higher granularity data from the Liquid Argon (LAr) electromagnetic calorimeter. For each LAr Trigger Tower, the eFEX receives data from 10 'supercells' in four layers, as shown in Figure 2.
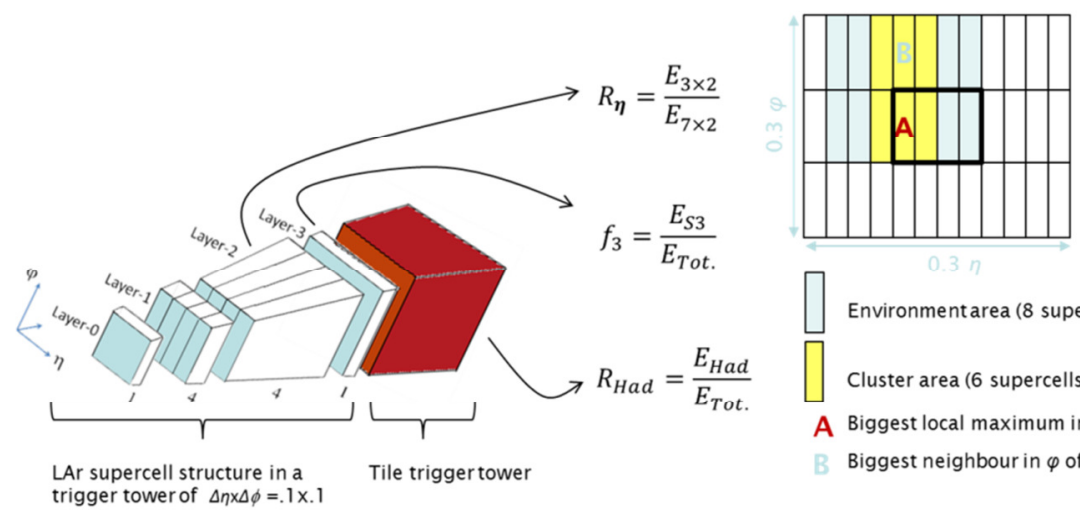

Environment area (8 supercells)

Cluster area (6 supercells)

A Biggest local maximum in $\eta$

Biggest neighbour in $\varphi$ of $A$

Figure 2. eFEX trigger algorithms [8] 
This makes it possible to increase the discriminatory power of L1Calo by running a collection of new trigger algorithms, including $R_{\eta}, f_{3}$ and $R_{\text {Had }}$, that analyse shower shapes. These algorithms run in a window of $0.3 \times 0.3(\eta \times \phi)$ that slides by 0.1 in both $\eta$ and $\phi$ (such that neighbouring instances of the window overlap).

Figure 3 shows the results of a simulation comparing the performance of the current (Run 2) algorithms with the eFEX (Phase I) algorithms. It shows the eFEX can reduce the EM trigger rate by a factor of $\sim 3$, or allow the trigger threshold to be lowered by $\sim 7 \mathrm{Gev}$ at the $20 \mathrm{KHz}$ reference point. Further optimization on eFEX algorithms is under study.

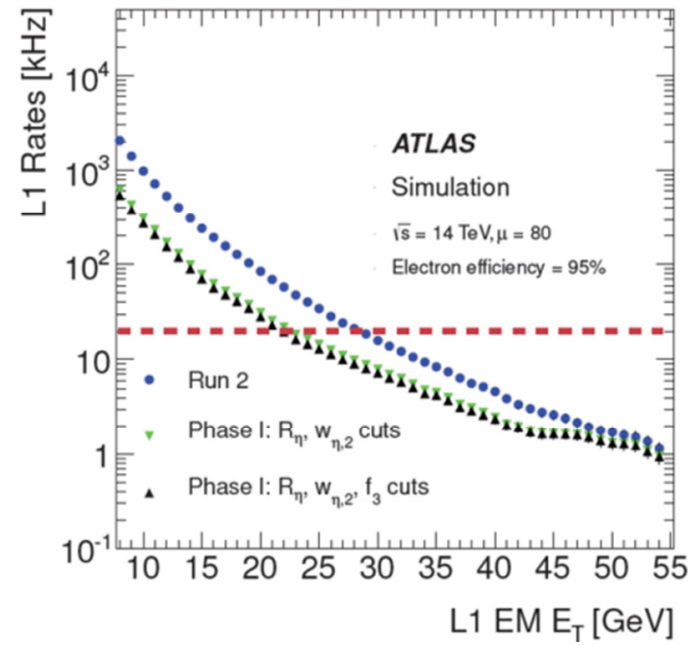

Figure 3. eFEX trigger performance [9]

The $\mathrm{jFEX}$ identifies jets, and calculates $\sum E_{T}$ and $E_{T}^{\text {miss }}$. In the current system, these functions are implemented by the JEP. The jFEX improves on the performance of the JEP by a number of means. It receives higher-granularity calorimeter data $(0.1 \times 0.1(\eta \times \phi)$ rather than $0.2 \times 0.2)$ and implements a Gaussian-weighted filter, giving it greater discriminatory power; it can implement a larger algorithm window; and each jFEX module processes data from a complete ring of the calorimeter in $\phi$, enabling in-time pileup suppression and improving the calculation of $\sum E_{T}$ and $E_{T}^{\text {miss }}$.

Figure 4 shows the results of simulations comparing the performance of the algorithms that can be implemented on the jFEX, with those currently run on the JEP. It shows how the turnon curves of the jFEX are sharper - a fact that can be used to raise the trigger thresholds without losing efficiency, leading to rate reductions similar to the eFEX.

\section{Prototype design}

\section{1 eFEX}

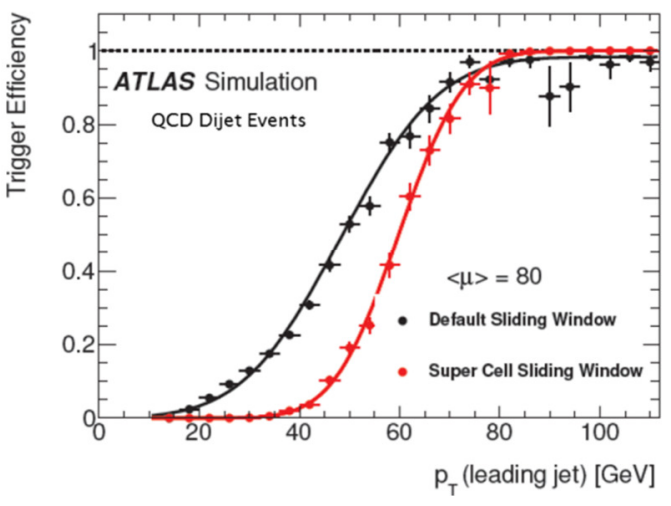

Figure 4. jFEX trigger performance [10]

\subsubsection{Processing Area}

The eFEX subsystem processes data from the calorimeters within the region of $2.5 \leq \eta \leq 2.5$ and $0 \leq \phi \leq 2 \pi-$ a total volume of $\sim 14 \mathrm{~Tb} / \mathrm{s}$. Given the limits of current technology, it is impossible to receive this into a single module. Due to the overlapping nature of the eFEX algorithm windows, partitioning the subsystem into multiple modules means a substantial volume of calorimeter data must be duplicated and/or shared between modules (and between FPGAs on the modules). This partitioning needs to balance the total number of modules, the number of FPGAs per module, the fibre count per module, the complexity of fibre mapping between the calorimeters and the eFEX, and the difficulty of sharing data between adjacent modules and between FPGAs. Figure 5 shows the partitioning of the eFEX prototype design. The middle eFEX module processes a core calorimeter area of $1.6 \times 0.8(\eta \times \phi)$, whereas 
the eFEX modules on two sides process a core calorimeter area of $1.7 \times 0.8(\eta \times \phi)$. Thus, three eFEX modules process a complete strip in the $\eta$ range, and 24 modules are required in total.

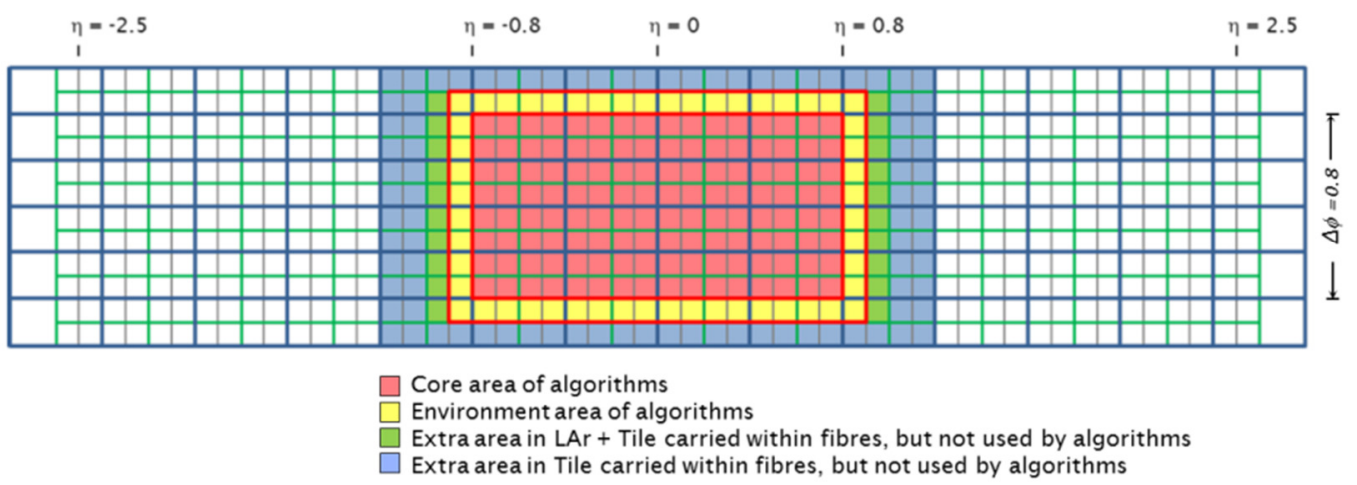

Figure 5. eFEX partitioning [11]

\subsection{2 eFEX prototype}

The eFEX prototype, shown in Figure 6, is an ATCA [12] module with a non-standard physical form: the front board is extended through Zone 3 into the rear shelf space to optimize the routing of the input fibres, which are connected to the module via a custom Rear Transition Module (RTM).

The eFEX PCB is a 22-layer board with six micro-via layers. It houses:

- 4 Xilinx Virtex-7 [13] FPGAs (XC7VX550T) for algorithm processing;

- 1 Xilinx Virtex-7 FPGA (XCVX330T) for control and readout functions;

- 17 Avago MiniPODs [14] for optical input (144 signals) and output (36);

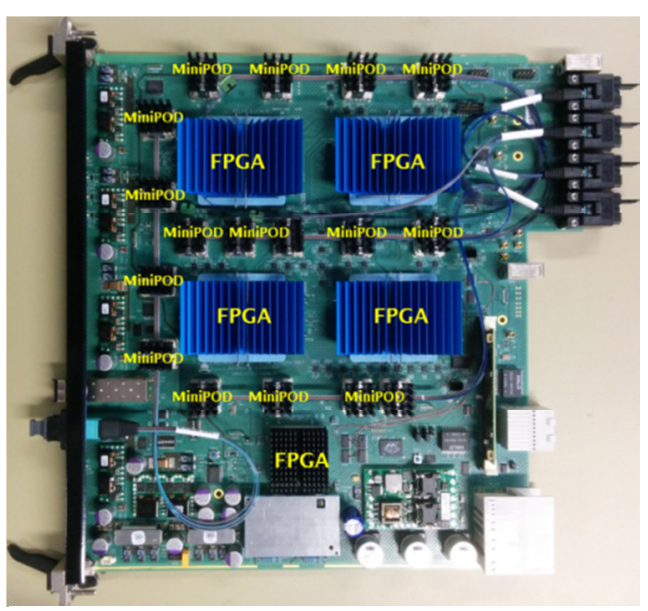

Figure 6. eFEX prototype

- 94 high-speed fan-out buffers (NB7VQ14M [15]) for data duplication between FPGAs.

The high-speed fan-out buffer NB7VQ14M was tested on a previous module, the HighSpeed Demonstrator (HSD) [16]. It exhibited very good signal quality at 10 Gbps with negligible propagation delay, and hence it was chosen for data duplication on the eFEX module. In total, there are about 450 high-speed multi-Gb/s differential tracks routed on a single eFEX. Blind and buried vias are used to achieve this density of signal tracks. The PCB is made from a low loss material (both Isola Itera and Megtron6 have been used on different prototype modules) and the PCB is rotated by $22^{\circ}$ to minimize the effect of PCB fibre glass weave on differential skew.

\section{2 jFEX}

\subsubsection{Processing area}

The jFEX receives data from the calorimeters within the region of $-4.9 \leq \eta \leq 4.9$ and $0 \leq \phi \leq 2 \pi-$ a total volume of $\sim 3 \mathrm{~Tb} / \mathrm{s}$. The jFEX subsystem is partitioned into 7 processing modules each covering a $\phi$ ring as shown in Figure 7. 
This $\phi$-ring coverage of each jFEX module enables it to calculate pile-up (i.e. energy density) for the $\eta$ range processed, and apply this as a correction to the jet and $E_{\mathrm{T}}{ }^{\text {miss }}$ algorithms.

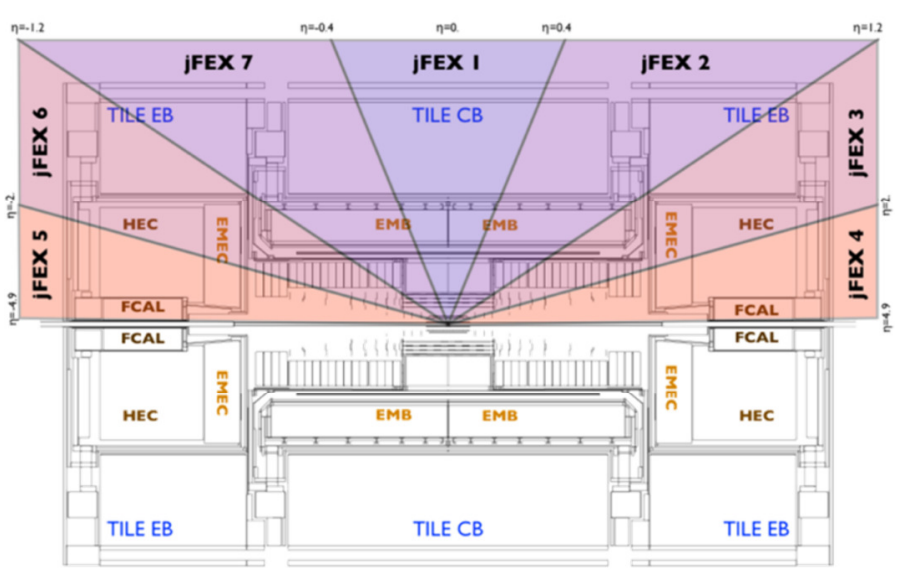

Figure 7. jFEX partitioning

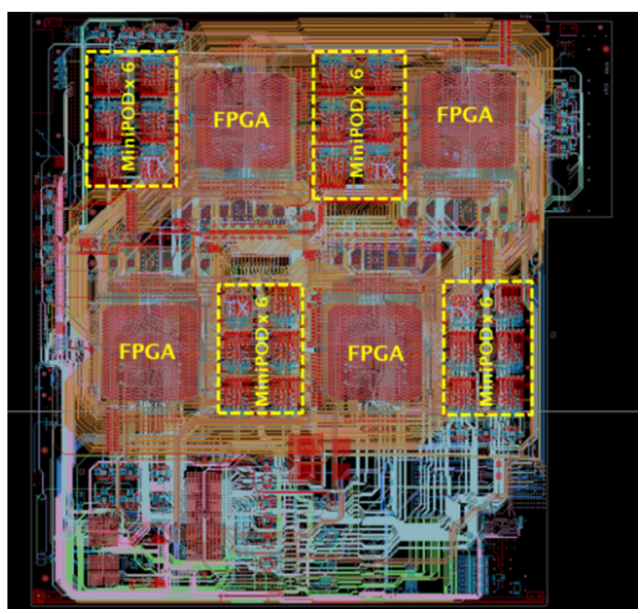

Figure 8. jFEX prototype PCB design

\subsection{2 jFEX prototype}

The jFEX prototype is currently being manufactured. The PCB layout is shown in Figure 8. It uses the same physical form factor as the eFEX, so that the modules can share the same RTM design. The jFEX PCB is implemented as a 24-layer board with 8 micro-via layers. It houses:

- 4 Xilinx Ultrascale [13] FPGAs (XCVU190) for algorithm processing and readout;

- 1 mezzanine card for control;

- 24 Avago MiniPODs for optical input (216 signals) and output (32);

Due to its larger algorithm window, the jFEX needs to share even more data between FPGAs than the eFEX. In total, there are about 540 high-speed multi-Gb/s differential tracks routed on a single jFEX PCB. A loopback feature of the Xilinx Multi-Gb/s Transceiver (MGT), Far-End PMA loopback, is used for data-sharing between FPGAs on the jFEX module. This makes use of the otherwise unused transmitters of MGTs with a small sacrifice of latency. Figure 9 shows the results of loopback tests done on a Xilinx Evaluation Board VCU110 (Ultrascale XCVU190), which shows a very good eye opening at $25 \mathrm{~Gb} / \mathrm{s}$.
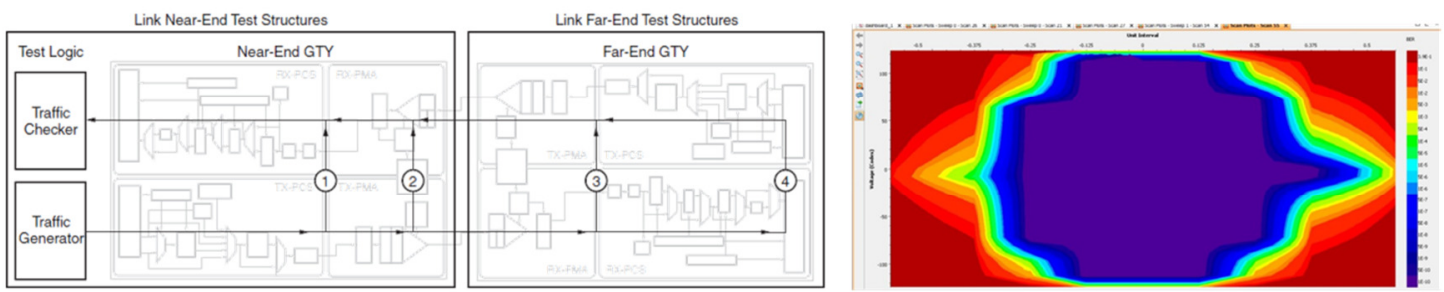

Figure 9. Xilinx MGT Far-End PMA loopback test (path 3 in diagram), IBERT 2-D Eye Scan 25Gb/s @ 10 ${ }^{-11}$ on Xilinx Evaluation Board VCU110 (Ultrascale XCVU190) 


\subsection{PCB design method}

The eFEX prototype and jFEX prototype share a lot of challenges in PCB design. Firstly, both are very high-density and high-speed PCB boards. The baseline speed of the inputs links specified in the ATLAS Phase-I TDR is $6.4 \mathrm{~Gb} / \mathrm{s}$. However, there is always strong desire to run the links faster in order to further improve trigger performance and flexibility. Secondly, both the eFEX and jFEX require complex channel mapping and data sharing. As a consequence, some high-speed links need to run very long signal tracks across the whole PCB between FPGAs. Thirdly, both the eFEX and jFEX have very high power consumption, approaching 400W per module. Cooling design will also be very challenging as all the power consumed turns into heat. To meet these challenges, the systematic PCB design method, which was developed with great success in the HSD project, has been adopted in both eFEX and jFEX PCB design. Notably, in addition to signal-integrity simulation, power-integrity simulation (as shown in Figure 10) is particularly important for these modules. The power rails for the FPGA cores and MGTs on both the eFEX and jFEX need to carry more than 100A current; the voltage drops across the power distribution networks thus become significant design constraints.

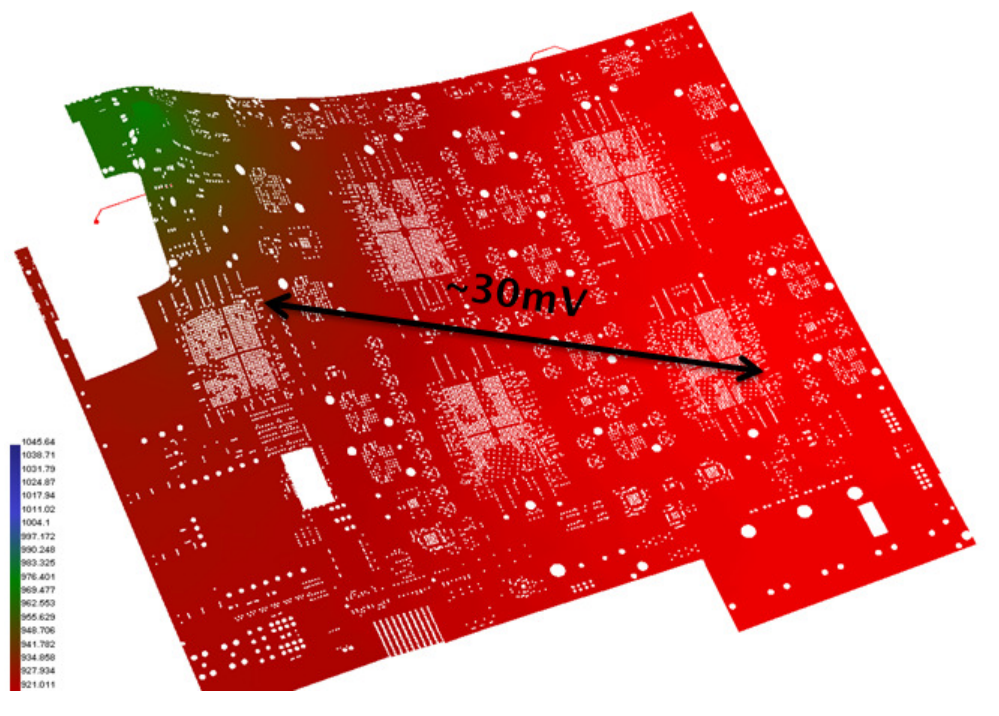

Figure 10. eFEX MGT power plane DC voltage drop simulation at 35A

\section{Prototype test}

After passing initial power-on and boundary scan tests smoothly, the eFEX prototype (Figure 6) was tested with the FTM and the LAr Digital Processing System (DPS) prototype, in a systematic check of all the eFEX high-speed input and output links.

In order to validate the TDR baseline link speed and test the upper limit of the possible link speeds, all eFEX high-speed links were tested at three different speeds $(6.4 \mathrm{~GB} / \mathrm{s}, 11.2 \mathrm{~Gb} / \mathrm{s}$ and $12.8 \mathrm{~Gb} / \mathrm{s})$. The decision on the link speed has a significant impact on the FEX architecture, especially for jFEX, where different link speeds required completely different partitioning.

The test setup for these link speed tests is very close to the final system as shown in Figure 1. For example, a FOX demonstrator was used to mimic the complex fibre mapping and insertion loss between the LAr DPS and the eFEX. 
For the link tests with the LAr DPS, the link sources were Altera Arria 10 FPGAs [17] with MGTs capable of up to $14 \mathrm{~Gb} / \mathrm{s}$. The first eFEX prototype is fitted with Xilinx speed grade -2 Virtex-7 FPGAs, with MGTs specified up to $11.3 \mathrm{~Gb} / \mathrm{s}$. The FTM is fitted with a Xilinx speed grade -3 Virtex-7 FPGAs, with MGTs specified up to $13.1 \mathrm{~Gb} / \mathrm{s}$.

\subsection{Link speed test results}

The test results obtained for the TDR baseline link speed of $6.4 \mathrm{~Gb} / \mathrm{s}$ are extremely good, with wide-open 2-D eye scans and Bit Error Rates of less than $10^{-}$ ${ }^{14}$ (no error over $3 \times 10^{14}$ bits) for 257 out of the 264 input links on the eFEX prototype.

At $11.2 \mathrm{~Gb} / \mathrm{s}$, the opening of the 2-D eye scans on the eFEX is still very good, as shown in Figure 11. Figure 12 shows the overall statistics of the open

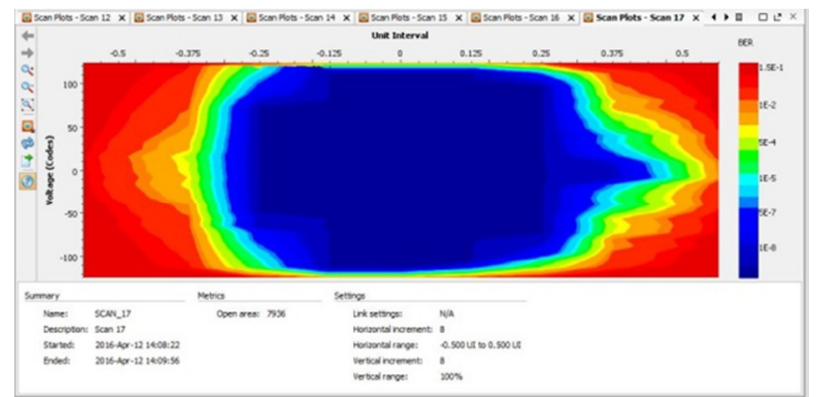

Figure 11. Typical eFEX input link 2-D eye-scan @ $11.2 \mathrm{~Gb} / \mathrm{s}$ areas of 2-D eye scans for all eFEX input links at $11.2 \mathrm{~Gb} / \mathrm{s}$. The Bit Error Rate on 257 out of 264 eFEX input channels is less than $10^{-14}$ (no error over $3 \times 10^{14}$ bits). Of the results for the other 7 links, 4 are correlated to less-optimal PCB routing (which can be improved in next PCB iteration), and 3 are due to a bad high-speed fan-out buffer (which can be repaired).

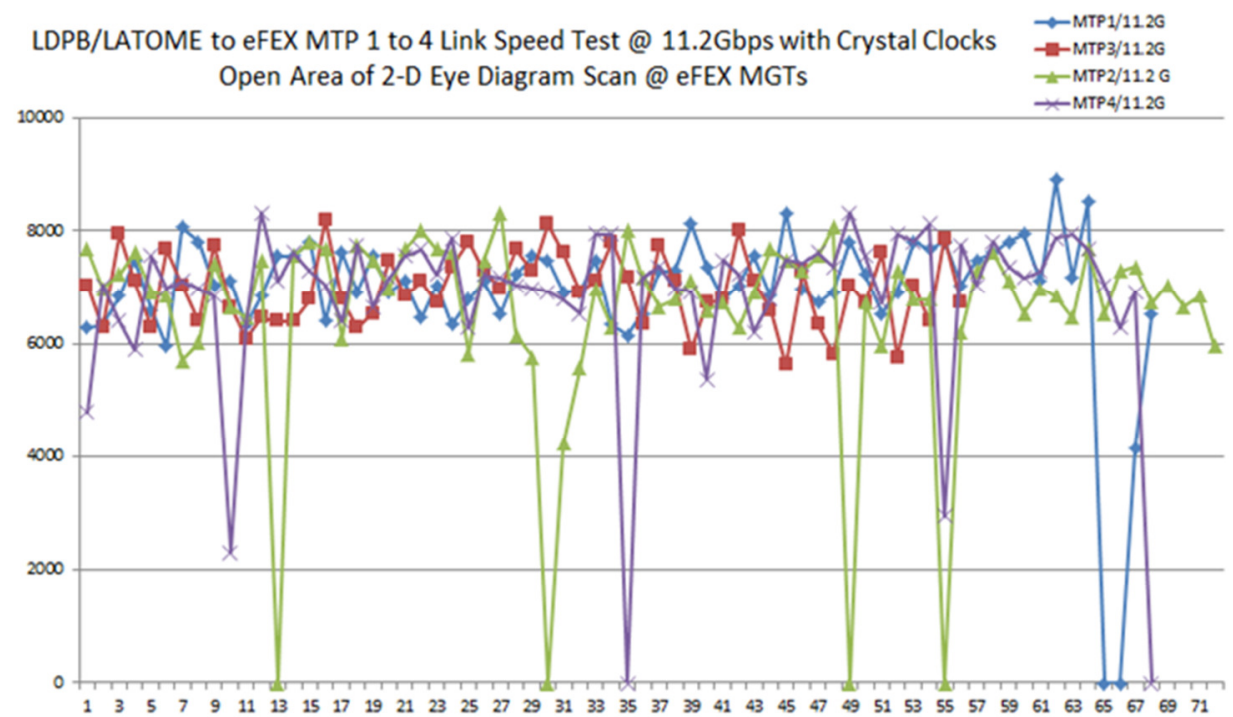

Figure 12. Open Area of 2-D eye scan for all eFEX input links

At $12.8 \mathrm{~Gb} / \mathrm{s}$, many links on the eFEX prototype still work, but a significant number fail, as this is outside FPGA MGT's specified speed range. In order to evaluate the eFEX performance at $12.8 \mathrm{~Gb} / \mathrm{s}$, another eFEX prototype will be fitted with Xilinx speed grade -3 Virtex-7 FPGA, with MGTs capable of running up to $13.1 \mathrm{~Gb} / \mathrm{s}$. 


\subsection{Link speed decision}

Based on the above excellent test results, and previous test results between the LAr DPS and the L1Calo gFEX, 11.2 Gb/s has been adopted as the new baseline link speed between LAr and L1Calo. This has greatly simplified the jFEX architecture, increased the dynamic range of the calorimeter data received by L1Calo, and simplified the link protocol, all of which improve L1Calo trigger performance.

\section{Conclusion}

The ATLAS Level-1 Calorimeter Trigger will be upgraded as part of the ATLAS Phase-I upgrades for 2019. Development of both the eFEX and jFEX are well underway, with prototypes under test or in manufacture. A systematic PCB design method, centred on PCB simulation and validation, has been used in developing these high-speed, high-density and highpower modules. The test results of the first eFEX prototype are very good, as a result of which the baseline for the speed of links into L1Calo has been increased from $6.4 \mathrm{~Gb} / \mathrm{s}$ to $11.2 \mathrm{~Gb} / \mathrm{s}$, simplifying the architecture and improving the performance of the trigger.

\section{References}

[1] Lyndon Evans and Philip Bryant, LHC Machine, 2008 JINST 3 S08001. http://iopscience.iop.org/1748-0221/3/08/S08001

[2] ATLAS collaboration, The ATLAS Experiment at the CERN Large Hadron Collider, 2008 JINST 3 S08003. http://iopscience.iop.org/1748-0221/3/08/S08003

[3] ATLAS collaboration, ATLAS Level-1 Trigger: TDR, CERN/LHCC/98-14. http://atlas.web.cern.ch/Atlas/GROUPS/DAQTRIG/TDR/tdr.html

[4] ATLAS collaboration, Technical Design Report for the Phase-I Upgrade of the ATLAS TDAQ System, CERN-LHCC-2013-018. https://cds.cern.ch/record/1602235/files/ATLAS-TDR-023.pdf

[5] R. Achenbach et al, The ATLAS Level-1 Calorimeter Trigger, 2008 JINST 3 P03001. http://iopscience.iop.org/1748-0221/3/03/P03001

[6] Weihao Wu et al, The development of the Global Feature Extractor for the LHC Run-3 upgrade of the L1 Calorimeter trigger system, ATL-DAQ-PROC-2016-010. https://cds.cern.ch/record/2162162/

[7] https://atlas.web.cern.ch/Atlas/GROUPS/PHYSICS/UPGRADE/CERN-LHCC-2013018/fig_19.png

[8] https://atlas.web.cern.ch/Atlas/GROUPS/PHYSICS/UPGRADE/CERN-LHCC-2013018/fig_20.png

[9] https://atlas.web.cern.ch/Atlas/GROUPS/PHYSICS/UPGRADE/CERN-LHCC-2013017/fig_11.png

[10] https://atlas.web.cern.ch/Atlas/GROUPS/PHYSICS/UPGRADE/CERN-LHCC-2013017/fig_14b.png

[11] https://atlas.web.cern.ch/Atlas/GROUPS/PHYSICS/UPGRADE/CERN-LHCC-2013018/fig_26.png 
[12] AdvancedTCA PCIMG 3.0 Short Form Specification http://www.picmg.org/pdf/PICMG_3_0_Shortform.pdf

262 [13] https://www.xilinx.com/products/silicon-devices/fpga.html

[14] MicroPOD ${ }^{\mathrm{TM}}$ and MiniPOD ${ }^{\mathrm{TM}}$ 120G Transmitters/Receivers http://www.avagotech.com/pages/minipod_micropod

[15] http://www.onsemi.com/PowerSolutions/product.do?id=NB7VQ14M

[16] W. Qian, ATLAS level-1 calorimeter trigger upgrade for phase-I, 2013 JINST 8 C01039. http://iopscience.iop.org/article/10.1088/1748-0221/8/01/C01039

[17] https://www.altera.com/products/fpga/arria-series/arria-10/overview.html 